\title{
Use and Indications of Human Acellular Dermis in Ventral Hernia Repair at a Community Hospital
}

\author{
William W. Hope, Devan Griner, Ashley Adams, W. Borden Hooks, and Thomas V. Clancy
}

South East Area Health Education Center, Department of Surgery, New Hanover Regional Medical Center, P.O. Box 9025, Wilmington, NC 28401, USA

Correspondence should be addressed to William W. Hope, william.hope@seahec.net

Received 21 August 2012; Accepted 11 September 2012

Academic Editor: Georg M. Huemer

Copyright (๑) 2012 William W. Hope et al. This is an open access article distributed under the Creative Commons Attribution License, which permits unrestricted use, distribution, and reproduction in any medium, provided the original work is properly cited.

\begin{abstract}
Background. To evaluate the use, indications, and short-term outcomes for human acellular dermis. Methods. We retrospectively reviewed patients having human acellular dermis placed for ventral hernia repair from January 2008 through October 2009. Demographic information, operative details, and outcomes of patients with and without recurrences were compared; a $P$ value $<0.05$ was considered significant. Results. 115 patients met inclusion criteria. The average age was 60 years (range, 24-89). The technique of repair included primary repair with overlay of mesh in $76 \%$, bridge repair in $13 \%$, and underlay in $11 \%$. Average cost of mesh per operation was $\$ 3,709$ (range $\$ 191-10,630$ ). Open repairs were performed in $90 \%$ of patients with addition of component separation in $12 \%$. At an average of 13 months, 58 patients were available for followup (50\%), with a $47 \%$ recurrence rate. The morbidity rate was $48 \%$ and the mortality rate was $2 \%$. Technique of repair was the only significant risk factor for recurrence with bridge repairs associated with a higher rate of recurrence $(P<0.05)$. Conclusions. The use of biologic grafts for ventral hernia repair is becoming more popular especially in clean cases. Although followup is limited, there remains a high recurrence rate associated with the use of human acellular dermis.
\end{abstract}

\section{Introduction}

Inguinal and ventral hernia repairs are some of the most common surgical procedures performed worldwide. Good evidence supports the concept of a tension-free repair in most cases with the use of mesh in inguinal and ventral hernia repairs [1-4]. Synthetic meshes, such as polypropylene, polyester, and expanded polytetrafluoroethylene (ePTFE) have been widely used and studied; however, concerns regarding infection have somewhat limited their use and efficacy.

The introduction of biologic grafts in 1999 revolutionized hernia repair and allowed surgeons to place these materials in infected fields. The theoretical advantage of these products for use in infected fields relates to their inherent properties of a regenerative matrix; however, robust scientific evidence supporting this is lacking [5]. Despite this, and largely due to early promising results, biologic grafts have been widely accepted by the surgical community as an alternative to synthetic products in contaminated and potentially infected fields. Increasing interest in the field of biologic mesh has resulted in a dramatic increase in the number and types of biologic products available, with over $\$ 400$ million spent on biologic grafts in the United States in 2007 [5]. This rapid increase in technology has made choosing the right mesh for each patient quite challenging to the surgeon, and indications for the use of biologic mesh are becoming blurred. Due to this and the paucity of data associated with many of the biologic products, we evaluated our experience with the use of human acellular dermis, one of the most commonly used biologic grafts, its indications, and outcomes for use in ventral hernia repair.

\section{Methods}

Following Institutional Review Board approval, we retrospectively reviewed all patients having human acellular dermis placed for repair of ventral hernias from January 1, 2008 through October 31, 2009. During the study period, there were no limitations on the use of biologic mesh 
in our hospital. Fifteen general surgeons and one plastic surgeon, with a varying degree of interest and expertise in hernia surgery, performed hernia repairs during the study period. Indications for use of the biologic mesh were left to the discretion of the surgeon. Demographic information, operative details, and outcomes were documented. Followup data were obtained from the patients' electronic medical records, and recurrence rates were obtained from physical exam or by imaging, specifically computed tomography (CT) scans. Patients with and without recurrences were compared using $t$-test, Wilcoxon rank sum, chi-squared, and Fisher's exact when appropriate; a $P$ value $<0.05$ was considered significant.

\section{Results}

During the study period, 115 patients met inclusion criteria. The average age was 60 years (range, $24-89$ years); $61 \%$ were female and $86 \%$ Caucasian. Biologic grafts were used in clean hernia repairs in $74 \%$ of cases. Alloderm (Life Cell Corp., Branchburg, NJ) was the human acellular dermis used in $79 \%$ of cases and FlexHD (Ethicon Inc., Somerville, NJ) was used in the remaining $21 \%$. The average operating room time was 125 minutes (range, 31-420 minutes). Recurrent hernias were present in $18 \%$ of patients, with 25 -month average time from the previous hernia repair to operation. In the patients with recurrent hernias, $19 \%$ had a previous mesh infection. The most common previous operation was open, and the most common mesh used was human acellular dermis (Table 1). A concomitant gastrointestinal or soft tissue surgery was performed in 23\% of patients (Table 2). The technique of repair included primary repair with overlay of mesh in $76 \%$, bridge repair in $13 \%$, and underlay in $11 \%$. No patients had repairs with the graft placed in the retromuscular space. The average cost of mesh per operation was \$3,709 (range \$191-\$10,630). Open repairs were performed in $90 \%$ of patients with addition of a standard open component separation with incision of the external oblique in $12 \%$. At an average 13 months, 58 patients (50\%) were available for followup and had a $47 \%$ recurrence rate. The morbidity rate was $48 \%$ (Table 3 ), and the mortality rate was $1.7 \%$. The overall wound infection rate was $14 \%$ with $3 \%$ of patients having to have their human acellular dermis removed and the remaining $11 \%$ able to be treated with antibiotics alone or antibiotics with local wound debridement and graft salvage. The technique of repair was the only significant risk factor for recurrence with bridge repairs associated with a higher rate of recurrence $(P<0.05)$ (Tables 4, 5, 6, and 7).

\section{Discussion}

Biologic grafts for use in complex hernia repair have been a major breakthrough in the field of hernia surgery. At present, there are 12 different biologic grafts commercially available, which differ based on many unique characteristics including the source material, processing techniques, and handling characteristics. Biologic grafts can initially be divided into heterograft, not from a human source, or allograft, from a cadaveric source.
TABle 1: Characteristics of the 26 patients who had a recurrent hernia operation with the use of acellular human dermis.

\begin{tabular}{lc}
\hline Type of most recent operation & \\
\hline Open & $76 \%$ \\
Lap & $19 \%$ \\
Unknown & $5 \%$ \\
\hline Previous mesh & $24 \%$ \\
\hline Human acellular dermis & $19 \%$ \\
ePTFE & $14 \%$ \\
Composite, bard/ventralex & $5 \%$ \\
Proceed surgical mesh & $10 \%$ \\
Polypropylene & $14 \%$ \\
Suture & $14 \%$ \\
Unknown &
\end{tabular}

ePTFE: expanded polytetrafluoroethylene.

TABLE 2: Type of concomitant surgery performed in patients undergoing hernia repair.

\begin{tabular}{lc}
\hline Associated cases & \\
Colostomy/ileostomy takedown & $3 \%$ \\
Small bowel resection & $8 \%$ \\
Nissen/foregut surgery & $3 \%$ \\
Colectomy & $6 \%$ \\
Above knee amputation & $1 \%$ \\
Cholecystectomy & $1 \%$ \\
Cystorrhaphy & $1 \%$ \\
None & $77 \%$ \\
\hline
\end{tabular}

TABLe 3: Type and percentage of morbidity following hernia repair.

\begin{tabular}{lc}
\hline Morbidity & Percentage \\
\hline None & $52 \%$ \\
Ileus & $20 \%$ \\
Urinary retention & $2 \%$ \\
Anemia & $3 \%$ \\
Atelectasis & $3 \%$ \\
Respiratory failure/insufficiency & $7 \%$ \\
Renal failure/insufficiency & $2 \%$ \\
Atrial fibrillation & $2 \%$ \\
Altered mental status/confusion & $2 \%$ \\
Wound infection & $11 \%$ \\
Infection requiring mesh removal & $3 \%$ \\
\hline
\end{tabular}

Human acellular dermis products are widely used and studied biologic products. Several cadaveric allografts are currently available including Alloderm (Life Cell Corporation, Branchburg, NJ), AlloMax (Tutogen Medical Inc, Alachua, FL), and FlexHD (Musculoskeletal Tissue Foundation, Edison, NJ). Alloderm has been the most often used, as it was the first product available, and many series have reported its use for the repair of complex abdominal wall reconstruction. Alloderm is created from cadaveric skin, is 
TABLE 4: Comparison of recurrence rates based on demographics and patient factors.

\begin{tabular}{lccc}
\hline Variable & Recurrent & Not recurrent & $P$ value \\
\hline $\begin{array}{l}\text { Age (years) } \\
\text { Gender }\end{array}$ & $62.8 \pm 13.9$ & $54.7 \pm 15.7$ & 0.5 \\
$\quad$ Male & $38 \%$ & $62 \%$ & \\
$\quad$ Female & $52 \%$ & $48 \%$ & 0.3 \\
Race & & & \\
$\quad$ White & $46 \%$ & $50 \%$ & 1.0 \\
Black & $54 \%$ & $50 \%$ & 0.053 \\
Incarcerated & $30 \%$ & $10 \%$ & \\
Type of hernia & & & \\
Incisional & $85 \%$ & $84 \%$ & 0.53 \\
Ventral & $15 \%$ & $10 \%$ & \\
Parastomal & $0 \%$ & $6 \%$ & \\
ASA & & & \\
1 & $0 \%$ & $3 \%$ & 0.9 \\
2 & $30 \%$ & $61 \%$ & \\
3 & $55 \%$ & $10 \%$ & \\
4 & $15 \%$ & $3 \%$ & \\
5 & $0 \%$ & & \\
\hline
\end{tabular}

TABLE 5: Comparisons of recurrence rate based on previous hernia surgery.

\begin{tabular}{lccc}
\hline Variable & Recurrence & $\begin{array}{c}\text { No } \\
\text { recurrence }\end{array}$ & $P$ value \\
\hline Recurrent & $26 \%$ & $23 \%$ & 0.8 \\
\hline Of recurrent cases: & & & \\
\hline Previous mesh infection & $29 \%$ & $14 \%$ & 1.0 \\
Type of most recent operation & & & \\
$\quad$ Open & $71 \%$ & $29 \%$ & 1.0 \\
$\quad$ Lap & $71 \%$ & $29 \%$ & \\
Previous mesh & & & \\
$\quad$ Human acellular dermis & $43 \%$ & $14 \%$ & \\
$\quad$ ePTFE & $43 \%$ & $14 \%$ & \\
$\quad$ PTFE/polypropylene & $14 \%$ & $14 \%$ & 0.4 \\
$\quad$ Polypropylene & $0 \%$ & $30 \%$ & \\
$\quad$ Suture & $0 \%$ & $14 \%$ & \\
$\quad$ Unknown & $0 \%$ & $14 \%$ & \\
\hline
\end{tabular}

ePTFE: polytetrafluoroethylene.

noncrosslinked, and freeze dried requiring approximately a 20- to 30-minute soak before use.

Initial reports on the use of Alloderm in complex hernias were promising. Three major centers reported on 171 patients with only a $4 \%$ graft removal rate and with an expected high rate of wound complications inherent in this patient population [6-8]. As these studies were preliminary reports, followup was short and difficult to interpret as were the techniques of repair. Further studies from several authors reported a laxity associated with the use of Alloderm, especially when this material was used as a "bridge" or
TABLE 6: Comparisons of recurrence rates based on class of case and thickness of graft.

\begin{tabular}{lccc}
\hline Variables & Recurrence & No recurrence & $P$ value \\
\hline Class & & & \\
$\quad$ Clean & $78 \%$ & $71 \%$ & \\
Clean-contaminated & $22 \%$ & $19 \%$ & 0.4 \\
$\quad$ Contaminated & $0 \%$ & $10 \%$ & \\
Removal Mesh/Infected & $0 \%$ & $6 \%$ & 0.5 \\
Thickness & & & \\
$\quad$ Normal $(<0.8 \mathrm{~mm})$ & $81 \%$ & $61 \%$ & \\
$\quad$ Intermediate $(0.8-1.7 \mathrm{~mm})$ & $4 \%$ & $0 \%$ & 0.06 \\
$\quad$ Ultrathick $(>1.8 \mathrm{~mm})$ & $15 \%$ & $39 \%$ & \\
\hline
\end{tabular}

TABLE 7: Comparisons of recurrence rate based on operative details.

\begin{tabular}{lccc}
\hline Variables & Recurrence & No recurrence & $P$ value \\
\hline Associated cases & $22 \%$ & $19 \%$ & 0.8 \\
Method of procedure & & & \\
$\quad$ Open & $78 \%$ & $87 \%$ & 0.4 \\
$\quad$ Laparoscopic & $11 \%$ & $0 \%$ & \\
$\quad$ Lap converted to open & $11 \%$ & $13 \%$ & \\
Component separation & $11 \%$ & $16 \%$ & 0.7 \\
Type of repair & & & \\
$\quad$ Primary closure w/overlay & $52 \%$ & $81 \%$ & \\
$\quad$ Bridge & $30 \%$ & $6 \%$ & $\mathbf{0 . 0 4 0}$ \\
$\quad$ Underlay & $18 \%$ & $13 \%$ & \\
Amount of mesh (cm ${ }^{2}$ ) & $108.3 \pm 94.5$ & $114.9 \pm 94.5$ & 0.6 \\
OR time (minutes) & $152.9 \pm 99.7$ & $119.4 \pm 88.6$ & 0.1 \\
Estimated blood loss (mL) & $71.85 \pm 85.1$ & $97.3 \pm 155.8$ & 1.0 \\
\hline
\end{tabular}

interposition repair. Blatnik and colleagues reported on 11 patients who underwent complex abdominal hernia repairs and reported $80 \%$ recurrence rate at a mean followup of 24 months [9]. Candage and colleagues reported on a larger series with a subset of 20 patients that underwent a bridge repair [10]. They reported a 30\% recurrence rate with $88 \%$ of the patients who developed eventration having a "bridge" repair. Bluebon-Langer and colleagues reported on 7 patients who underwent component separation with an interposition repair with Alloderm, observing the laxity within 12 months [11]. Jin and colleagues also reported on the effect that technique of repair has on outcomes in 37 patients undergoing abdominal wall repair with Alloderm showing that it should be used only as reinforcement after primary fascial reapproximation [12]. Based on these reports, it was evident that technique of repair had an important impact on the recurrence rate associated with these biologic materials. Our findings echo these reports in that the technique of repair was the only significant risk factor for recurrence. The "bridge" repairs had a significantly higher recurrence rate than onlay or underlay techniques. While the high recurrence rate associated with bridging repairs is well known today and has been previously reported [12], most of our bridging repairs were performed early in our experience and show the unrealized high expectations that these surgeons had for 
these new biologic products. In our bridging repairs only 1 patient had it placed as a temporary abdominal closure technique.

Our series is unique to previously published reports in many ways. First, our report is from a community teaching hospital comprised of private practitioners and academic surgeons differing from many previous reports from large trauma centers or specialized hernia centers that have a specific focus on abdominal wall reconstruction. Second, a surprising finding of our study is that the majority of cases in which the biologic graft was used were clean cases, whereas the majority of prior studies have been in complex or infected hernia repairs, because this was the accepted initial indication for these products. Interestingly, we report 6 cases where human acellular dermis was used in a recurrent hernia where the previous mesh was human acellular dermis. In review of these cases, 5 of the cases were clean with one being contaminated. Only one of the cases had a different surgeon performing the first and second operation and in this case case the first surgeon used Alloderm and the second surgeon used FlexHD. The rationale for repeat use of the biologic is unknown and difficult to explain with 3 of these 6 patients having a documented repeat recurrence. Our report shows that with increased experience with these products, surgeons are liberalizing the indications for the use despite little supporting scientific literature.

One potential drawback of these biologic products is the cost. Several studies have evaluated the cost associated with the use of these products recommending caution in their use until further studies and information are available $[5,9]$. Several hospitals have also limited the use of these products to "hernia specialist" or for complex/infected cases. During this study, our hospital had no constraints on the use of biologic products; however, it had limitations relating to the types of biologic products available. One impetus for this study was to evaluate our use of these products and the economic impact for our hospital. Although no direct comparisons were made, the cost of biologic mesh for a clean case far exceeds that for a synthetic mesh at our hospital; however, we cannot comment further on the economic impact as we did not evaluate hernia repairs using synthetic mesh. Cost comparisons are also very difficult due to the followup needed to evaluate for long-term complications such as mesh infections and recurrences.

There are several limitations to our study. One is that the indication for use of biologic mesh at our community hospital was surgeon dependent and this has caused liberalization of use that may not be seen in other hospitals. It is evident that our surgeons incorrectly bought into the premise that these human acellular grafts would be as strong as synthetic mesh and could be used in clean and infected fields without the possibility of infections. Secondly, our followup is fairly limited due to the nature of multiple different surgeons, from multiple different groups, with different followup patterns performing these operations and this could affect our recurrence rate. Although our followup is limited, our series reports a high recurrence rate, especially for a series with many clean cases and likely with improved followup our recurrence rate may be even higher. One caveat to our recurrence rate is that recurrences were often found on CT scans obtained for indications other than to evaluate for recurrent hernias, and many of these recurrences were asymptomatic, so patients did not undergo further surgery. Despite this, based on our surgeons' experience and appreciation of this high recurrence rate, many surgeons in our hospital have begun to use a porcine dermis product for ventral hernia repairs, as it is believed to result in less laxity and stretching. Technique of repair has also been discussed with a near abandonment of the "bridge" repair except in extremely complex cases where temporary closure is needed and increasing use of component separation.

In conclusion, we report on the use of acellular human dermis for ventral hernia repair including results from many surgeons using different techniques with a high rate of usage in clean cases. Our results show a high recurrence rate associated with their use and that the technique of repair significantly impacts recurrence rates with the interposition or "bridge" repairs resulting in a significantly higher rate compared with an underlay or overlay technique. Based on our surgeons experiences the use of human acellular dermis in clean ventral hernia repairs should be abandoned secondary to the unacceptable high recurrence rate and increased cost. Secondary to our results, our hospital has shifted to using different biologic products for ventral hernia repair and have put more emphasis on outcomes associated with these products.

\section{Disclosure}

Data from this study were presented as an invited oral presentation at the Annual Hernia Repair, March 16-19, 2011, San Francisco, CA.

\section{Conflict of Interests}

Drs. W. W. Hope, D. Griner, W. B. Hooks, and T. V. Clancy and Ms. A. Adams have no commercial associations or financial disclosures that might pose or create a conflict of interests with information presented in this paper.

\section{References}

[1] N. W. Scott, K. McCormack, P. Graham, P. M. Go, S. J. Ross, and A. M. Grant, "Open mesh versus non-mesh for repair of femoral and inguinal hernia," Cochrane Database of Systematic Reviews, no. 4, Article ID CD002197, 2002.

[2] J. W. A. Burger, R. W. Luijendijk, W. C. J. Hop et al., "Longterm follow-up of a randomized controlled trial of suture versus mesh repair of incisional hernia," Annals of Surgery, vol. 240, no. 4, pp. 578-585, 2004.

[3] W. W. Vrijland, M. P. van den Tol, R. W. Luijendijk et al., "Randomized clinical trial of non-mesh ver u mesh repair of primary inguinal hernia," British Journal of Surgery, vol. 89, no. 3, pp. 293-297, 2002.

[4] R. W. Luijendijk, W. C. J. Hop, M. P. van den Tol et al., "A comparison of suture repair with mesh repair for incisional hernia," The New England Journal of Medicine, vol. 343, no. 6, pp. 392-398, 2000. 
[5] M. J. Rosen, "Biologic mesh for abdominal wall reconstruction: a critical appraisal," American Surgeon, vol. 76, no. 1, pp. $1-6,2010$.

[6] J. J. Diaz Jr., J. Guy, M. B. Berkes, O. Guillamondegui, and R. S. Miller, "Acellular dermal allograft for ventral hernia repair in the compromised surgical field," American Surgeon, vol. 72, no. 12, pp. 1181-1187, 2006.

[7] J. H. Patton Jr., S. Berry, and K. A. Kralovich, "Use of human acellular dermal matrix in complex and contaminated abdominal wall reconstructions," American Journal of Surgery, vol. 193, no. 3, pp. 360-363, 2007.

[8] H. Kim, K. Bruen, and D. Vargo, "Acellular dermal matrix in the management of high-risk abdominal wall defects," American Journal of Surgery, vol. 192, no. 6, pp. 705-709, 2006.

[9] J. Blatnik, J. Jin, and M. Rosen, "Abdominal hernia repair with bridging acellular dermal matrix-an expensive hernia sac," American Journal of Surgery, vol. 196, no. 1, pp. 47-50, 2008.

[10] R. Candage, K. Jones, F. A. Luchette, J. M. Sinacore, D. Vandevender, and R. L. Reed, "Use of human acellular dermal matrix for hernia repair: friend or foe?" Surgery, vol. 144, no. 4, pp. 703-711, 2008.

[11] R. Bluebond-Langner, E. S. Keifa, S. Mithani, G. V. Bochicchio, T. Scalea, and E. D. Rodriguez, "Recurrent abdominal laxity following interpositional human acellular dermal matrix," Annals of Plastic Surgery, vol. 60, no. 1, pp. 76-80, 2008.

[12] J. Jin, M. J. Rosen, J. Blatnik et al., "Use of acellular dermal matrix for complicated ventral hernia repair: does technique affect outcomes?" Journal of the American College of Surgeons, vol. 205, no. 5, pp. 654-660, 2007. 


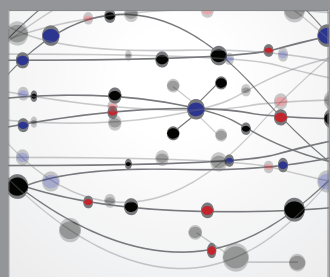

The Scientific World Journal
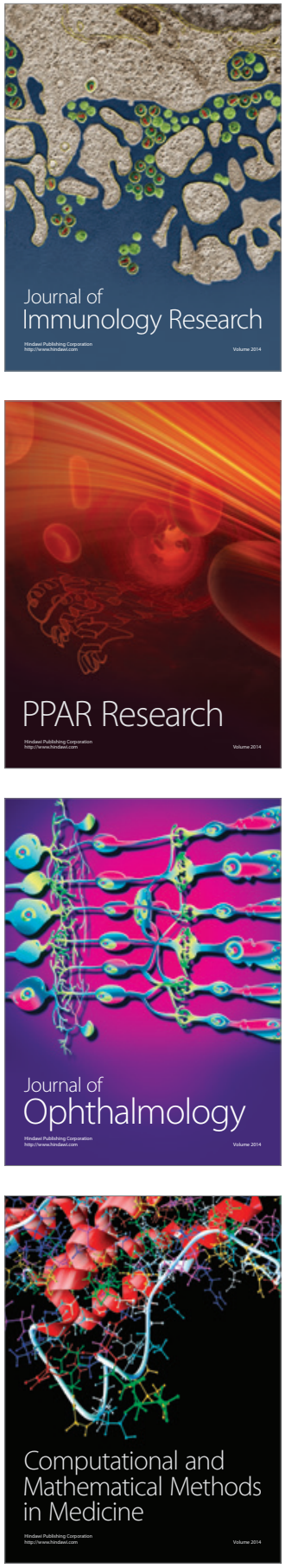

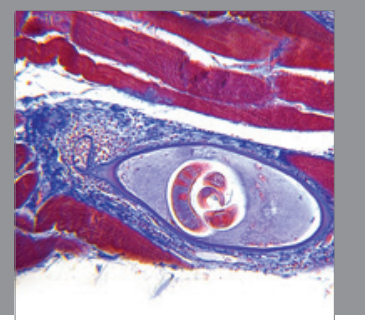

Gastroenterology

Research and Practice
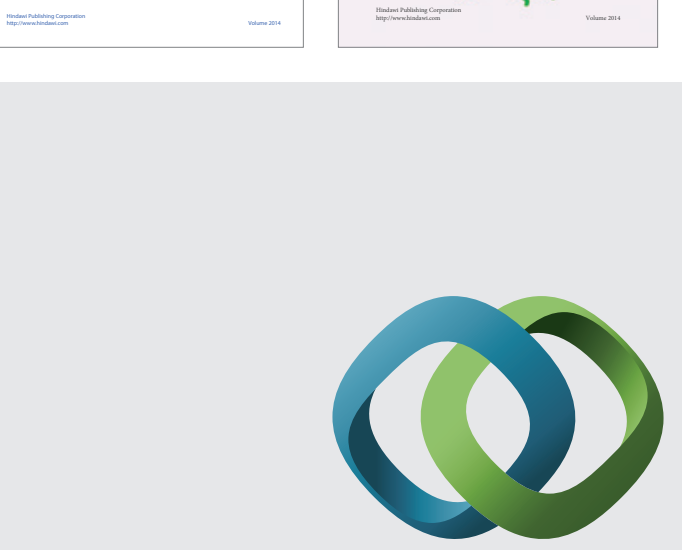

\section{Hindawi}

Submit your manuscripts at

http://www.hindawi.com
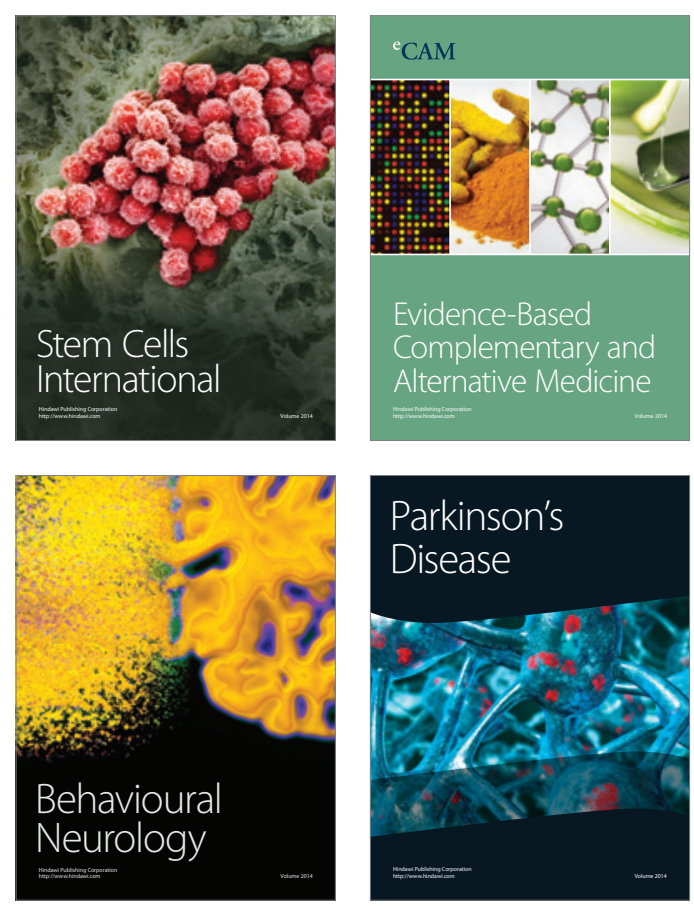

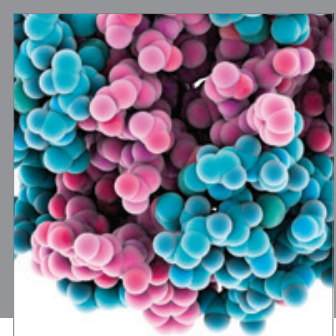

Journal of
Diabetes Research

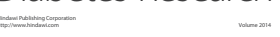

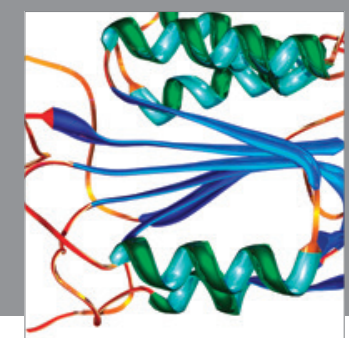

Disease Markers
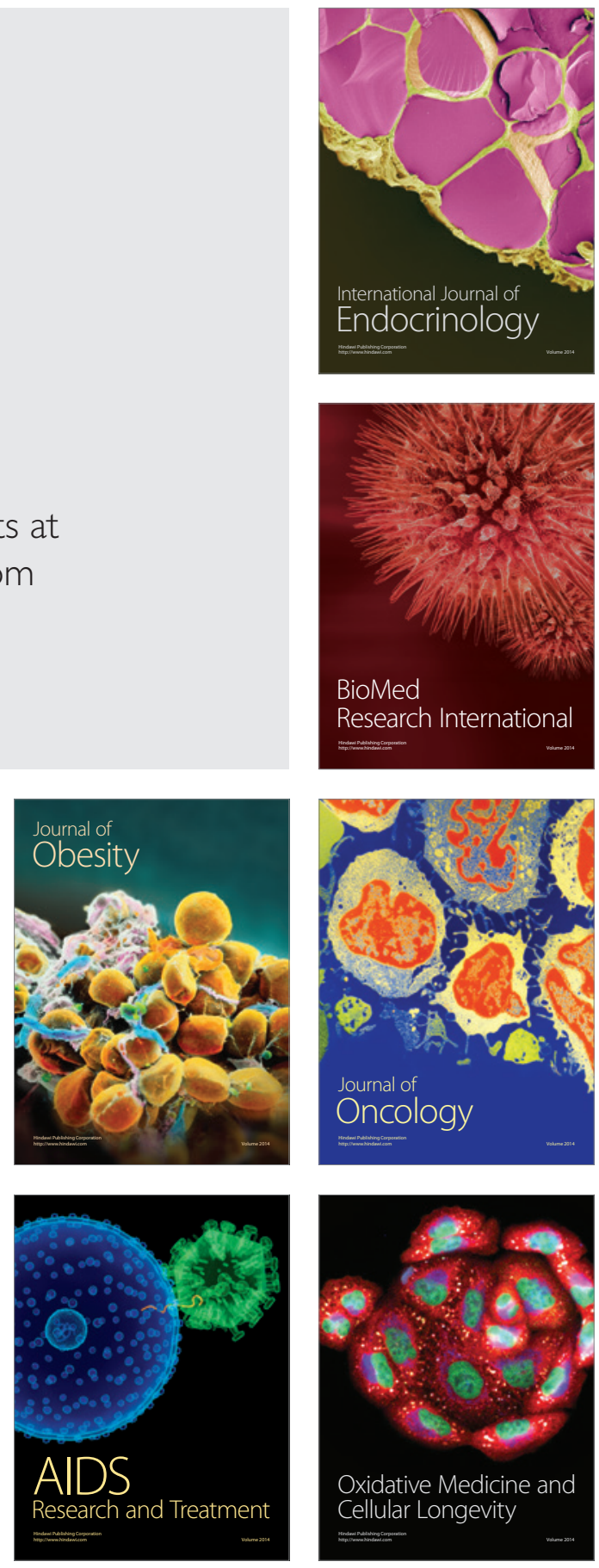